\title{
Jugular lymphatic sacs in the first trimester of pregnancy: the prevalence and the potential value in screening for chromosomal abnormalities
}

\author{
Peter N. van Heesch*, Pieter C. Struijk, Helen \\ Brandenburg, Eric A. Steegers and Hajo \\ I. Wildschut
}

Erasmus MC, University Medical Center Rotterdam Rotterdam, The Netherlands

\begin{abstract}
Objective: To investigate the prevalence of detectable jugular lymphatic sacs in a setting for first trimester screening for Down syndrome, and to evaluate the influence of jugular lymphatic sacs on the screening performance for chromosomal abnormalities.

Methods: A prospective single center study (Erasmus University Medical Center, Rotterdam, The Netherlands) over a period of one year (January 2003-February 2004). First trimester nuchal translucency measurement was performed in a study population of 415 fetuses. Additionally, the transversal plane with the spine and mandible was visualized to verify the presence of jugular lymphatic sacs. The jugular lymphatic sacs were measured anterior-posterior. The association between nuchal translucency and jugular lymphatic sacs was tested.

Results: Follow-up was complete in 406 cases (97.8\%). Jugular lymphatic sacs could be visualized in 98 out of 415 (23.5\%). The nuchal translucency thickness and the mean of the left and right jugular lymphatic sac were significantly correlated.

Conclusion: The sonographic visualization of jugular lymphatic sacs significantly predicts chromosomal abnormalities, although nuchal translucency is a better predictor. Nuchal translucency and jugular lymphatic sacs are strongly correlated and therefore not applicable in a combination test.
\end{abstract}

Keywords: Chromosomal abnormalities; first trimester screening; jugular lymphatic sacs; nuchal translucency; sonographic marker.

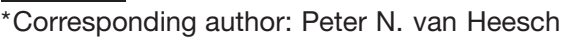
Department of Obstetrics and Gynecology

Division of Obstetrics and Prenatal Medicine

Erasmus University Medical Center Rotterdam

Room He-115

PO Box 2040

3000 CA Rotterdam

The Netherlands

Tel.: + 31107032390

E-mail: p.vanheesch@erasmusmc.nl
}

\section{Introduction}

First trimester measurement of nuchal translucency (NT) with maternal blood sampling is standard antenatal care in the Netherlands. This screening strategy is a sensitive method for the identification of fetuses at risk of aneuploidy $[13,18]$ and is associated with increased risk of genetic syndromes and fetal structural malformations $[14,15]$. Although many theories have been proposed, a common morphogenesis explaining the interrelationship between the complete spectrum of fetal malformations and enlarged nuchal translucency is still lacking. Previous studies demonstrated abnormal developed jugular lymphatic sacs (JLS) in combination with an enlarged NT [1, 5-7]. Since a disturbance in the lymphangiogenesis precedes the development of an increased NT [5, 7], it is hypothesized that JLS size could be an earlier and better predictor of chromosomal abnormalities.

We conducted an observational study to investigate the prevalence of detectable JLS and to evaluate the test performance by adding this parameter to first trimester screening. This study was integrated in a setting for first trimester screening for Down syndrome (DS).

\section{Methods}

During a period of 13 months (January 9, 2003-February 26, 2004), an observational study was performed. All women with singleton pregnancies who attended the department of Obstetrics and Prenatal Medicine of the Erasmus University Medical Center for first trimester screening were informed about the study. Patients were included in this study after giving oral informed consent. Ultrasound examinations of 415 fetuses were carried out between the 11 and 14 weeks' gestation. Scans were preformed with an ATL HDI-3000 ultrasound system (Advanced Technology Laboratories, Seattle, WA, USA) either transvaginally, using a $7-\mathrm{MHz}$ probe or abdominally, using a $5-\mathrm{MHz}$ probe. Gestational age was derived on the basis of the first day of the last menstrual period (LMP) and confirmed by ultrasound assessment of fetal biometry. The ultrasound examinations were carried out following the strict methodological criteria set by the Fetal Medicine Foundation (FMF) ${ }^{\circledR}[10,12]$. An experienced and specially trained FMF-certified ultrasonographer $(\mathrm{PH})$ conducted all first trimester ultrasound examinations. The NT was measured in the mid-sagittal section of the fetus as the maximum thickness of the subcutaneous translucency between the soft tissue overlaying the cervical spine and the skin. The presence and size of the jugular lymphatic sacs were investigated in a 
transversal plane through the mandible and spinal cord and confirmed in sagittal and coronal planes, where feasible. Measurements of the anterior-posterior size of the JLS were performed in the transversal plane as shown in Figure 1. Digital recordings of the first trimester screening and a transverse plane of the neck, including mandible and spinal cord and the presence or absence of the jugular lymphatic sacs, were collected for further analysis. In all women, first trimester screening with additional fetal biometry and visualization, measurement and documentation of jugular lymphatic sacs were completed within a time frame of $30 \mathrm{~min}$. Invasive procedures, i.e., transabdominal chorionic villius sampling at 11-14 weeks' gestation or amniocentesis at 15-19 weeks' gestation, were performed, where indicated. Follow-up was obtained by means of questionnaires, which were returned to us by the obstetrician or midwife supervising delivery.

\section{Statistical analysis}

If JLS could not be visualized, the size was set to zero. The nonparametric Kendall's Tau-b test, that takes ties into account, was done to measure the association between NT and JLS size. In the subset where JLS size is larger than zero, the Pearson cor-

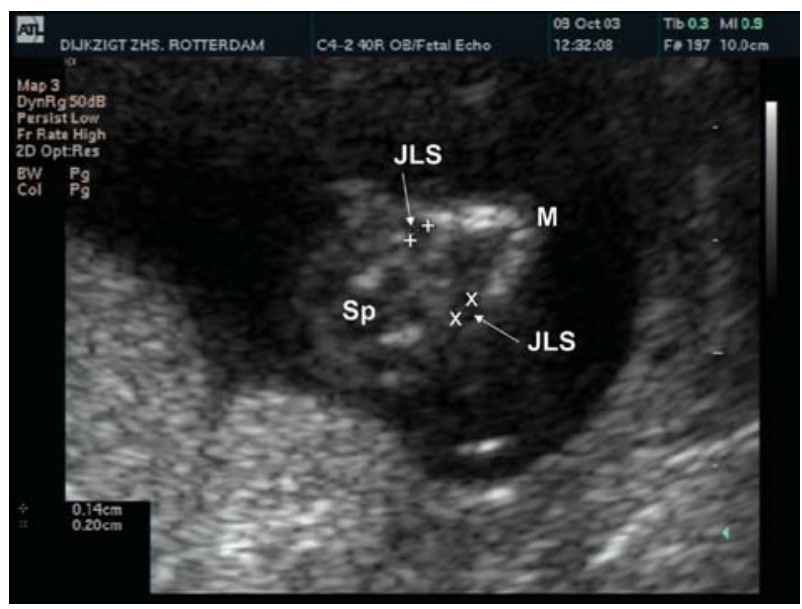

Figure 1 Transversal plane through the mandible $(\mathrm{M})$ and spinal cord (Sp). Bilateral normal size jugular lymphatic sac (JLS). Measurement of JLS is done in an anterior-posterior direction. relation coefficient between NT and the logarithm of JLS size was determined.

Statistical significance was defined as a $\mathrm{P}<0.05$. In the study group the risk of chromosomal abnormalities was modelled using binary logistic regression. Statistically significant effects and interactions were identified by backward stepwise elimination using the likelihood ratio test. The probability criterion for stepwise entry was set at 0.05 and for removal from the equation at 0.1 . The statistical analyses were performed using the SPSS ${ }^{\circledR}$ statistical package for Windows release 11.1 (SPSS Inc., Chicago, IL).

\section{Results}

During the study period, a total of 415 women with a viable singleton pregnancy were evaluated. Median maternal age in the total study population was 36 years (range 18-43 years). The study population was mainly white, i.e., 95\% Caucasian, 1.2\% Asian and 3.8\% AfroAmerican. As a result of various pleas and reminders, complete follow up was obtained in 406 women (study group). In the latter group 13 chromosomal abnormalities (3.2\%) were detected, while adverse pregnancy outcome (fetal loss, fetal death and neonatal death) was documented in 9 other cases (2.2\%), as summarized in Tables 1 and 2 .

NT was successfully evaluated in all fetuses. There were no differences between the groups with regard to maternal age, ethnicity and gestational age at examination. Median gestational age was $12+3$ weeks (range, $11+0$ weeks $-13+6$ weeks) and median $\mathrm{CRL}$ was $61.7 \mathrm{~mm}$ (range, 45-84 mm). After excluding the chromosomal abnormalities and adverse pregnancy outcome, a subset group of $384(94.6 \%)$ women with an uncomplicated pregnancy were included for final analyses.

In this subset the $95^{\text {th }}$ percentile for NT size was $2.5 \mathrm{~mm}$. Women were therefore considered "test-positive" if the NT size was above this level. The JLS were detectable in $23 \%$ (97/406) women of the study group. In the same subset of 384 uncomplicated pregnancies

Table 1 Chromosomal abnormalities.

\begin{tabular}{|c|c|c|c|c|c|c|c|}
\hline & $\mathrm{n}$ & Gestational age & NT & JLS left & JLS right & Test NT & Test JLS \\
\hline \multirow[t]{6}{*}{ Trisomy 21} & 7 & $12+5$ & 3.1 & 2.1 & 2.9 & + & + \\
\hline & & $12+1$ & 4.5 & 1.4 & 1.7 & + & - \\
\hline & & $12+4$ & 4.6 & 4.3 & 6.4 & + & + \\
\hline & & $13+0$ & 4.8 & 1.9 & 2.4 & + & + \\
\hline & & $13+6$ & 5.2 & 15.5 & 14.6 & + & + \\
\hline & & $13+2$ & 5.7 & 0.0 & 0.0 & + & - \\
\hline \multirow[t]{3}{*}{ Trisomy 18} & 3 & $11+2$ & 5.3 & 6.6 & 6.1 & + & + \\
\hline & & $12+3$ & 6.0 & 3.4 & 2.8 & + & + \\
\hline & & $13+4$ & 4.3 & 1.7 & 1.3 & + & - \\
\hline Trisomy 13 & 1 & $12+4$ & 1.6 & 0.0 & 0.0 & - & - \\
\hline Monosomy X & 1 & $11+0$ & 9.2 & 12.9 & 9.1 & + & + \\
\hline $47 X Y Y$ & 1 & $12+2$ & 2.0 & 1.9 & 1.7 & - & - \\
\hline
\end{tabular}


Table 2 Adverse pregnancy outcome.

\begin{tabular}{|c|c|c|c|c|c|c|c|}
\hline & $\mathrm{n}$ & Gestational age & NT & JLS left & JLS right & Test NT & Test JLS \\
\hline \multirow[t]{5}{*}{ Early fetal loss ( $<20$ weeks) } & 5 & $11+3$ & 2.0 & 0.0 & 0.0 & - & - \\
\hline & & $13+5$ & 1.0 & 0.0 & 0.0 & - & - \\
\hline & & $13+1$ & 0.8 & 0.0 & 0.0 & - & - \\
\hline & & $13+6$ & 1.0 & 0.0 & 0.0 & - & - \\
\hline & & $13+3$ & 1.0 & 0.0 & 0.0 & - & - \\
\hline \multirow[t]{2}{*}{ Intra uterine fetal death } & 2 & $13+5^{*}$ & 3.8 & 0.0 & 0.0 & + & - \\
\hline & & $13+6$ & 1.9 & 0.0 & 0.0 & - & - \\
\hline \multirow[t]{2}{*}{ Neonatal death } & 2 & $12+1$ & 1.5 & 0.0 & 0.0 & - & - \\
\hline & & $13+5^{\star \star}$ & 2.3 & 2.8 & 2.9 & - & + \\
\hline
\end{tabular}

*Second pregnancy. First pregnancy hydrops fetalis, TOP at 19 weeks.

${ }^{\star \star}$ Complex heart defect.

the $95^{\text {th }}$ percentile for the mean left and right JLS size was $2.0 \mathrm{~mm}$. Women were therefore considered "testpositive" if the JLS size was above this level. Median size of detectable JLS size was $1.7 \mathrm{~mm}$ (range $0.7-15.5 \mathrm{~mm}$ ). Table 1 shows that in the group of chromosomal abnormalities, all seven fetuses with trisomy 21 tested positive for NT and 5/7 for JLS. For trisomy 18, all fetuses also tested positive for NT and 2/3 for JLS. The fetus with monosomy $X$ (Turner syndrome, TS) tested positive in both tests. Because of hypoplasia of the lymphatic system in fetuses with TS [17], it is questionable whether these should be included in our study. However, during the ultrasound investigation, the karyotype was not yet known to the investigator during the examination and the septated hygroma colli was identified as an enlarged NT accompanied with bilateral enlarged JLS. Although NT can identify about $80 \%$ of the fetuses with trisomy 13 in a regular first trimester screening program [13], in our study one fetus with Patau syndrome was tested negative by NT and JLS (Table 1). On ultrasound, however, dextrocardia was observed, which classified the fetus as at increased risk of chromosomal abnormality. An abnormal karyotype of $47 \mathrm{XYY}$ is not associated with enlarged NT and was tested negative by both NT and JLS. In the group of adverse pregnancy outcome (Table 2) post mortem reports on the early fetal loss were not available. In the study group of 406 fetuses, a significant non-parametric correlation (Kendall's Tau-b, $R=0.129$ ) was found between NT and JLS $(\mathrm{P}<0.01)$. As shown in Figure 2, the subset of fetuses in which the mean left and right JLS size was larger than zero $(n=97)$, showed a strong correlation between NT and the logarithm of JLS. The Pearson correlation coefficient was 0.636 and the P-value was highly significant $(P<0.001)$. The binary regres- sion results are shown in Table 3. In this study group, NT in combination with maternal age and JLS did not contribute to a statistically better prediction of chromosomal abnormalities than NT alone. Moreover, as enlarged NT and JLS are strongly interrelated, these parameters cannot be considered independent and therefore not applicable in a multiple parameter binary logistic regression analysis. Although both NT and JLS parameters are statistically significant predictors, the sensitivity for NT was better than for JLS.

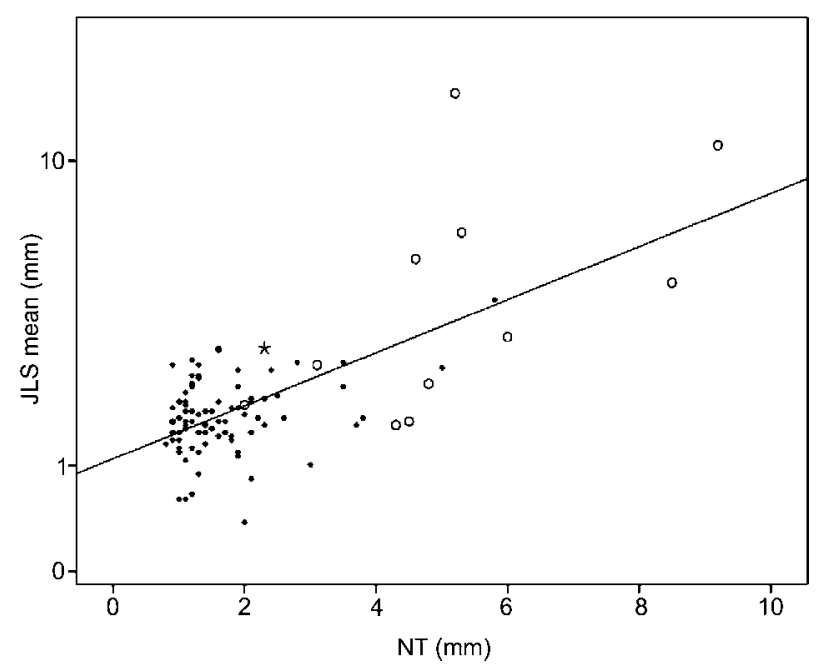

Figure 2 Correlation between NT and the mean JLS in the subset of the study group, where measured JLS size is larger than zero: *, refers to the pregnancy with visible JLS and adverse outcome due to the complex heart defect (Table 2); $\bullet$, uncomplicated pregnancy outcome; $\bigcirc$, chromosomal abnormality.

Table 3 Binary logistic regression results for chromosomal abnormalities.

\begin{tabular}{lllllll}
\hline & Threshold $^{*}(\mathrm{~mm})$ & Sensitivity (\%) & Specificity (\%) & Odds ratio & 95\% Confidence interval \\
\hline JLS & 2.0 & 62 & 95 & $3.81^{\star \star}$ & 2.19 & 6.64 \\
NT & 2.5 & 85 & 95 & $5.53^{\star \star}$ & 3.12 & 9.82 \\
\hline
\end{tabular}

*Women were considered screened positive if the distance was above this level.

** Statistically significant at the level $\mathrm{P}<0.001$. 


\section{Discussion}

This study was carried out to evaluate the prevalence of JLS and was integrated in a setting of first trimester screening for Down syndrome (DS) in order to evaluate its value in screening for chromosomal abnormalities. The association between NT, JLS and fetal outcome were investigated, but in this context, the prerequisite for adding JLS to first trimester measurement of NT should be an independent association with chromosomal abnormalities.

Nuchal translucency is a subcutaneous fluid accumulation in the neck region of unknown origin. However, Haak et al. [6, 7] showed that in mouse embryos with trisomy 16 the development of the JLS preceded the NT and that the JLS showed distension just prior to the occurrence of the increased NT. This notion was supported by other investigators [1, 2, 4, 5]. Trisomy 16 mouse embryos are considered to be the animal model for trisomy 21 in the human $[8,9,11,16]$. The delayed organization and connection of these JLS to the venous circulation might explain the transient nature of the NT. Human studies on NT and JLS [1-4, 6, 7] were unable to give conclusive evidence on the question if JLS precedes NT or vice versa. It merely suggests that there seems to be a fetus-specific pattern in the development of the jugular lymphatic system, and therefore a unique expanding phase of the jugular lymphatic sacs.

If the development of JLS in human fetuses is similar to the animal model of the trisomy 16 mouse embryo [1, 2, 4-7], further analysis might determine its possibility as an early marker for chromosomal abnormalities before the $11^{\text {th }}$ week of gestation. Considering the large number of attrition of chromosomal abnormal pregnancies and pregnancies with major anomalies during the first trimester, this additional value remains to be discussed. The absence of JLS in early pregnancy could perhaps be used as a sonographic marker for ruling out chromosomal abnormalities (negative predictive value). In our study, the smallest JLS size detectable by ultrasound was $0.7 \mathrm{~mm}$, which approximated the spatial resolution of the ultrasound equipment in combination with the $7 \mathrm{MHz}$ transvaginal and $5 \mathrm{MHz}$ (broadband) abdominal transducers. In fact, the ability to detect JLS is limited by the spatial resolution of ultrasound machines. Although one could hypothesize that improvement of ultrasound imaging performance might improve the visualization of small JLS, their potential additional value for the early detection of fetal chromosomal abnormalities merits further research. Nevertheless, the sonographic visualization of JLS smaller than $2.0 \mathrm{~mm}$ is time consuming and requires specially trained and highly skilled ultrasound operators.

From our study we conclude that clearly visualized JLS significantly predict chromosomal abnormalities, although NT is a better predictor. In terms of test performance, however, the additional value of combined testing is limited as both predictors are interrelated.

\section{References}

[1] Bekker MN. From lymphatic development to nuchal translucency. PhD thesis. Amsterdam: Buiten and Schipperheijn; 2007.

[2] Bekker MN, Haak MC, Rekoert-Hollander M, Twisk J, Van Vugt JM. Increased nuchal translucency and distended jugular lymphatic sacs on first-trimester ultrasound. Ultrasound Obstet Gynecol. 2005;25:239-45.

[3] Bekker MN, Twisk JW, Bartelings MM, Gittenberger-de Groot AC, van Vugt JM. Temporal relationship between increased nuchal translucency and enlarged jugular lymphatic sac. Obstet Gynecol. 2006;108:846-53.

[4] Bekker MN, van den Akker NM, Bartelings MM, Arkesteijn JB, Fischer SG, Polman JA, et al. Nuchal edema and venous-lymphatic phenotype disturbance in human fetuses and mouse embryos with aneuploidy. J Soc Gynecol Investig. 2006;13:209-16.

[5] Gittenberger-De Groot AC, Van Den Akker NM, Bartelings MM, Webb S, Van Vugt JM, Haak MC. Abnormal lymphatic development in trisomy 16 mouse embryos precedes nuchal edema. Dev Dyn. 2004; 230:378-84.

[6] Haak MC. Nuchal translucency and cardiac failure. PhD thesis. Wageningen: Ponsen and Looijen; 2003.

[7] Haak MC, Bartelings MM, Jackson DG, Webb S, van Vugt JM, Gittenberger-de Groot AC. Increased nuchal translucency is associated with jugular lymphatic distension. Hum Reprod. 2002;17:1086-92.

[8] Holtzman DM, Bayney RM, Li YW, Khosrovi H, Berger CN, Epstein CJ, et al. Dysregulation of gene expression in mouse trisomy 16, an animal model of Down syndrome. Embo J. 1992;11:619-27.

[9] Miyabara S, Gropp A, Winking H. Trisomy 16 in the mouse fetus associated with generalized edema and cardiovascular and urinary tract anomalies. Teratology. 1982;25: 369-80.

[10] Pandya PP, Santiago C, Snijders RJ, Nicolaides KH. First trimester fetal nuchal translucency. Curr Opin Obstet Gynecol. 1995;7:95-102.

[11] Reeves RH, Irving NG, Moran TH, Wohn A, Kitt C, Sisodia $\mathrm{SS}$, et al. A mouse model for Down syndrome exhibits learning and behaviour deficits. Nat Genet. 1995;11: 177-84.

[12] Snijders RJ, Noble P, Sebire N, Souka A, Nicolaides KH. UK multicentre project on assessment of risk of trisomy 21 by maternal age and fetal nuchal-translucency thickness at 10-14 weeks of gestation. Fetal Medicine Foundation First Trimester Screening Group. Lancet. 1998; 352:343-6.

[13] Snijders RJ, Sebire NJ, Nayar R, Souka A, Nicolaides KH. Increased nuchal translucency in trisomy 13 fetuses at 10-14 weeks of gestation. Am J Med Genet. 1999;86: 205-7.

[14] Souka AP, Snijders RJ, Novakov A, Soares W, Nicolaides $\mathrm{KH}$. Defects and syndromes in chromosomally normal fetuses with increased nuchal translucency thickness at 10-14 weeks of gestation. Ultrasound Obstet Gynecol. 1998;11:391-400.

[15] Souka AP, Von Kaisenberg CS, Hyett JA, Sonek JD, Nicolaides $\mathrm{KH}$. Increased nuchal translucency with normal karyotype. Am J Obstet Gynecol. 2005;192:1005-21.

[16] von Kaisenberg CS, Krenn V, Ludwig M, Nicolaides KH, Brand-Saberi B. Morphological classification of nuchal skin in human fetuses with trisomy 21,18 , and 13 at $12-18$ 
weeks and in a trisomy 16 mouse. Anat Embryol (Berl). 1998;197:105-24.

[17] von Kaisenberg CS, Nicolaides KH, Brand-Saberi B. Lymphatic vessel hypoplasia in fetuses with Turner syndrome. Hum Reprod. 1999;14:823-6.

[18] Zoppi MA, Ibba RM, Putzolu M, Floris M, Monni G. Assessment of risk for chromosomal abnormalities at
10-14 weeks of gestation by nuchal translucency and maternal age in 5,210 fetuses at a single centre. Fetal Diagn Ther. 2000;15:170-3.

Received March 3, 2008. Revised May 27, 2008. Accepted June 5, 2008. Previously published online August 6, 2008. 